\title{
Wirkung visueller und auditiver Hinweise auf die visuelle Aufmerksamkeit und Lernergebnisse beim Einsatz physikalischer Lernvideos
}

\author{
Bianca Watzka (D) Christoph Hoyer (D) B Bernhard Ertl (iD • \\ Raimund Girwidz iD
}

Eingegangen: 15. November 2019 / Überarbeitet: 8. März 2021 / Angenommen: 12. Mai 2021 / Online publiziert: 15. Juni 2021

(C) Der/die Autor(en) 2021

Zusammenfassung Lernende haben oft Schwierigkeiten, Informationen aus visuellen, dynamischen Lernmedien zu extrahieren. Daher werden diese häufig durch Hinweise ergänzt, welche die visuelle Aufmerksamkeit steuern und den Lernerfolg verbessern können.

Bisher ist offen, wie gut der Lernerfolg beim Lernen mit Videos in Abhängigkeit von der Hinweismodalität vorhergesagt werden kann und welche Rolle dabei die visuelle Aufmerksamkeit spielt. Das Ziel dieser Studie ist, die Hinweismodalität zu variieren, um den Lernerfolg zu beeinflussen und dabei zu untersuchen, wie viel der so erzeugten Varianzen im Lernerfolg durch die visuelle Aufmerksamkeit erklärt werden können.

Dazu wurden zwei Videos von Wirbelstromexperimenten erstellt und mit Hinweisen versehen, die entweder visuell als Kombination aus Textfeldern und Spotlights oder auditiv als gesprochener Text implementiert waren. 40 Lehramtsstudierende wurden zufällig der visuellen oder auditiven Bedingung zugeteilt. Ein Eye-Tracker zeichnete die Blickbewegungen auf. Die Vor- und Nachtestung erfolgte durch Fragebögen, die affektive und kognitive Lernfaktoren erfassten.

Verglichen mit Textfeldern und Spotlights führten gesprochene Hinweise zu einer höheren visuellen Aufmerksamkeit und zu besseren Lernergebnissen. Allerdings förderten Textfelder und Spotlights die Anwendung der Fachsprache besser als gesprochene Hinweise. Bemerkenswert dabei ist, dass die visuelle Aufmerksamkeit bei den Lernergebnissen auf das durch den gesprochenen Hinweis adressierte Expe-

Bianca Watzka $(\bowtie) \cdot$ Christoph Hoyer $\cdot$ Raimund Girwidz

Lehrstuhl für Didaktik der Physik, Ludwig-Maximilians-Universität München,

Theresienstraße 37, 80333 München, Deutschland

E-Mail: Bianca.Watzka@1mu.de

Bernhard Ertl

Fakultät für Humanwissenschaften, Professur für Lernen und Lehren mit Medien, Universität der

Bundeswehr, Werner-Heisenberg-Weg 39, 85577 Neubiberg, Deutschland 
riment und somit auf das Videomaterial bezogen war. Im Gegensatz dazu war beim Erwerb der Fachtermini die visuelle Aufmerksamkeit auf die Textfelder und somit auf die Hinweise ausschlaggebend.

Aus den Ergebnissen lässt sich für die Gestaltung von Demonstrationsvideos ableiten, dass die Hinweismodalität auf die Lernziele abgestimmt werden sollte.

Schlüsselwörter Videos $\cdot$ Signalisierungsprinzip $\cdot$ Modalitätsprinzip $\cdot$ Visuelle Hinweise · Auditive Hinweise · Eye-Tracking · Physik · Experimente

\title{
Understanding the impact of visual and auditive cues on visual attention and learning outcomes while learning with physics videos
}

\begin{abstract}
Discovering relevant information in visual representations often provides difficulties for learners. Cues could complement such representations and thereby facilitate learning. The impact of the cues' modality on the mental processing of such representations is still unknown. Therefore, this study aims at investigating to what extent auditory and visual cues influence learners' visual attention and how far this predicts differences in learning outcomes.

The study implemented two videos about eddy currents with either auditory or visual cues. The visual cues comprised written texts as well as learners' attention focusing by highlighting AOIs. The auditory cues comprised spoken text that directed learners to the relevant elements. 40 pre-service teachers of physics participated in this study and were randomly assigned to the conditions. An eye-tracker recorded their eye-movements. Before and after this intervention, all participants passed a paper and pencil pre- and post-test that included several measures.

Data analysis comprised a mediation model that included visual attention. Participants with auditory cues gazed significantly longer and more often to the experiment shown in the video compared to participants with visual cues; they were also able to rehearse correctly significantly more items when describing and sketching the eddy current experimental settings. The visual attention on the video were able to explain the largest amount of variances with respect to the rehearsal of items.

Results of the study suggest complementing visual physical representations by auditory or visual cues with respect to the learning goals. Such facilitation would allow learners to perceive relevant information more often and longer and support thereby their rehearsal.
\end{abstract}

Keywords Cueing $\cdot$ Signaling $\cdot$ Modality $\cdot$ Eye-Tracking $\cdot$ Physics $\cdot$ Experimental videos

\section{Einleitung}

Die vorliegende Studie beschäftigt sich mit dem Lernen mit Demonstrationsvideos zu physikalischen Standardexperimenten im Rahmen der universitären Lehrerbildung. In Anlehnung an das Signalisierungsprinzip sind Hinweise in Videos eingebunden, um direkt während des Lernprozesses Schwierigkeiten aufgrund mangeln- 
der Vorkenntnisse zu kompensieren und Probleme mit der visuellen Wahrnehmung zu mildern. Das Modalitätsprinzip gibt Empfehlungen, wie die Hinweise und das Lernmaterial insgesamt dargestellt sein sollten, um Split-Attention-Effekte zu vermeiden und das Lernen zu unterstützen. So sollten gemäß dem Modalitätsprinzip zu bildhaften Darstellungen verbale Erläuterung eher gesprochen als geschrieben angeboten werden. Dass diese Regel nicht pauschal gilt, zeigt eine Studie von Glaser und Schwan (2020). Sie zeigen u. a. den besonderen Nutzen visueller Hinweise beim Interpretieren von Bildern für die Fähigkeit, sich an den Ort bestimmter Elemente im Bild zu erinnern. Die Wirkung der Hinweise ist demnach auch von den verfolgten Lernzielen abhängig.

Noch ist nicht abschließend geklärt, wie sich die Hinweismodalität in einem aus Bildinformationen bestehenden Lernmaterial wie Demonstrationsvideos auf die Fähigkeit auswirkt, verschiedene Lerninhalte wie Fachsprache, experimentelles Wissen etc. wiedergeben zu können und welche Rolle dabei die visuelle Aufmerksamkeit einnimmt.

Diese Studie trägt zur Aufdeckung eines möglichen Mediationsmechanismus bei, bei dem die visuelle Aufmerksamkeit die Wirkung der Hinweise auf den Lernerfolg mediiert. Die vorliegende Arbeit kann grundlegende Erkenntnisse über spezifische Stärken visueller bzw. auditiver Hinweise in dynamischen Lernmaterialien aufzeigen und Konsequenzen für die Konzeption solcher Lernmaterialien ableiten.

\section{Theoretischer Hintergrund}

\subsection{Dynamische Darstellungen als Lernmaterial}

Höffler und Leutner (2007) bestätigen in ihrer Metaanalyse, dass dynamische Darstellungen (hier: Animationen) dann ein besseres Lehrmittel als statische Bilder sind, wenn sich die dargestellte Dynamik direkt auf den Lerngegenstand bezieht. Auch für den Erwerb prozedural-motorischer Fähigkeiten können Animationen vorteilhaft sein (Höffler und Leutner 2007). Berney und Bétrancourt (2016) weisen in einer weiteren Metaanalyse die Überlegenheit von dynamischen Darstellungen (hier: Videos) gegenüber statischen Bildern nach. Jedoch heben sie hervor, dass diese generelle Überlegenheit unabhängig von den vermittelten Wissensarten ist. Obwohl die Ergebnisse nicht explizit zeigen, dass dynamische Darstellungen für verschiedene Wissensarten unterschiedlich gut geeignet sind, fordern Lowe und Schnotz (2014) sowie Berney und Bétrancourt (2016), dass näher darauf einzugehen ist, wann, warum und für wen dynamische Darstellungen lernförderlich sein können.

Der Gegenstand dieser Studie sind Demonstrationsvideos von Standardexperimenten, die den dynamischen Darstellungen zugeordnet werden können. Demonstrationsvideos sind eine Unterart von Lernvideos (Persike 2019) und werden eingesetzt, um Vorgehensweisen wie beispielsweise den Gebrauch eines Gegenstandes anzuleiten (Persike 2019) oder um ,,best practices“ zu präsentieren (Gaudin und Chaliès 2015). Demonstrationsvideos von Standardexperimenten eignen sich für das Zeigen prototypischer Aufbauten und Durchführungsszenarien (vgl. Schweinberger und Girwidz 2017). Insbesondere können sie in der universitären Lehrerbildung 
reale Experimentalübungen ergänzen, indem sie Gelegenheiten zur vorbereitenden Übung einer eigenen Versuchsdemonstration bieten. Auch wenn solche Videos einen methodischen Nutzen in der Lehre haben, ist anzunehmen, dass sie bezüglich des Lernerfolgs nicht per se anderen Medien überlegen sind. So ist bei Videos die Informationswahrnehmung erschwert, da sie in der Regel nur zeitlich begrenzt sichtbar sind. Hinzu kommen Probleme, die sich aus dem Absinken der Aufmerksamkeit (vgl. Loviscach 2019) und dem ,lean back“-Effekt, also dem passiven Konsumieren eines Videos ergeben (Arnold et al. 2013).

\subsection{Kognitive Theorie multimedialen Lernens (CTML)}

Zur Untersuchung von Hinweisen in Videos ist die CTML (Mayer 2014) zu berücksichtigen. Die Theorie erklärt auf drei Annahmen basierend, wie multimedial präsentierte Inhalte mental verarbeitet werden.

1. Die erste Annahme beschreibt, dass das multimediale Lernen über je einen visuellen und auditiven Verarbeitungskanal erfolgt.

2. Zweitens wird angenommen, dass beide Kanäle eine begrenzte Kapazität aufweisen. Demnach können Lernende pro Kanal nur eine bestimmte Anzahl an Informationen gleichzeitig verarbeiten.

3. Die dritte Annahme betrifft das Lernen als aktiven und konstruktiven Prozess. Dabei stehen drei kognitive Aktivitäten im Vordergrund. Beim Selektieren werden relevante visuelle und auditive Informationen ausgewählt und im jeweiligen Kanal mental verarbeitet. Das Organisieren beschreibt die Konstruktion von jeweils einem verbalen und bildhaften mentalen Modell, in dem Informationen durch Repräsentanten abgebildet sind. Beim Integrieren werden die beiden mentalen Modelle unter Einbezug des Vorwissens miteinander verbunden.

\subsubsection{Modalitätsprinzip}

Das Modalitätsprinzip setzt an der ersten und zweiten Annahme der CTML an (siehe 2.2). Es drückt allgemein aus, dass es für das Lernen von Vorteil ist, wenn zu grafisch präsentierten Informationen der begleitende Text gesprochen statt schriftlich dargeboten wird. Das Modalitätsprinzip wirkt sich u. a. positiv auf die visuelle Aufmerksamkeit aus, da sie nicht zwischen beiden Informationsquellen aufgeteilt werden muss, also kein Split-Attention-Effekt zu befürchten ist (vgl. Schmidt-Weigand et al. 2010).

In einer 43 Studien umfassenden Metaanalyse bestätigt Ginns (2005) mit mittlerer Effektstärke von $d=0,72$ den Modalitätseffekt und zeigt, dass Lernmaterialien mit Visualisierungen und gesprochenen Texten im Allgemeinen zu besseren Lernergebnissen führen als Lernmaterialien mit Visualisierungen und geschriebenen Texten.

Die lernförderliche Wirkung des Modalitätseffekts erklärt sich aus einer effektiveren Nutzung der Arbeitsspeicherkapazität, da die Informationsverarbeitung auf den visuellen und den auditiven Kanal verteilt wird. Entsprechend können mehr kognitive Ressourcen für die Verarbeitung des Lerninhalts genutzt werden und die Lernleistung steigt (Sweller et al. 2011). 
Allerdings können verschiedene Faktoren den Modalitätseffekt moderieren. Einschränkend kann gemäß Lindow et al. (2011) die Länge und Komplexität des Textes wirken. Begründet wird dies mit der Transienz von mündlichen bzw. Persistenz von geschriebenen Texten. Mündliche Texte sind nur vorübergehend präsent und müssen im Arbeitsgedächtnis behalten werden. Insbesondere bei langen oder komplexen mündlichen Texten könne es dadurch zu einer kognitiven Überlastung kommen, sodass sich der Modalitätseffekt bei diesen Texten auch umkehren könnte.

Das Modalitätsprinzip bezieht sich zwar nur auf verbale und bildhafte Informationen in Lernmaterialien. Es ist aber wahrscheinlich, dass auch die Modalität von Hinweisen in bildbasierten Lernmaterialien den Lernerfolg beeinflusst. Im nächsten Abschnitt wird die Verwendung aufmerksamkeitssteuernder Hinweise aus dem Signalisierungsprinzip der CTML motiviert und Bezug auf das Modalitätsprinzip genommen.

\subsubsection{Signalisierungsprinzip}

Das Signalisierungsprinzip setzt an der dritten Annahme der CTML (vgl. 2.2) an und besagt allgemein, dass es lernförderlich ist, wenn die Prozesse Selektieren, Organisieren und Integrieren durch in das Lernmaterial eingebettete Hinweise unterstützt werden (van Gog 2014). Hinweise können nach verschiedenen Kriterien kategorisiert werden. Eine Unterscheidung nach der Modalität führt zu den Kategorien visuell und auditiv. Beispiele für visuelle Hinweise sind u.a. farbliche Hervorhebungen, Textfelder, Spotlights, Pfeile oder Filmtechniken wie Zoomen. Auditive Hinweise können als gesprochene Texte gegeben werden.

In Metaanalysen bestätigen sowohl Richter et al. (2016) als auch Schneider et al. (2018) die lernförderliche Wirkung des Signalisierungsprinzips. Die Analyse von Richter et al. (2016) umfasst 27 Studien zum Signalisierungsprinzip. Als zentrale Erkenntnisse halten sie fest, dass sich Hinweise in multimedialen Lernmaterialien mit kleiner bis mittlerer Effektstärke positiv auf die Lernleistung auswirkt und dass vor allem Lernende mit niedrigem Vorwissen von den Hinweisen profitieren. Die Analyse von Schneider et al. (2018) umfasst 103 Studien zum Signalisierungseffekt und schließt auch Eye-Tracking-Daten mit ein. Auch diese Autoren bestätigen zusammengefasst die förderliche Wirkung des Signalisierungsprinzips auf den Lernerfolg. Sie zeigen auch, dass das Signalisierungsprinzip die kognitive Belastung zu reduzieren scheint und sich wahrscheinlich positiv auf der motivationalen Ebene auswirkt. Außerdem scheinen aufmerksamkeitssteuernde Hinweise in multimedialen Materialien mit kleiner bis mittlerer Effektstärke längere Lernzeiten im Allgemeinen und längere Blickdauern auf relevante Informationen im Speziellen zu bewirken (Schneider et al. 2018). Im Gegensatz zur Analyse von Richter et al. (2016) deuten die Ergebnisse von Schneider et al. (2018) nicht darauf hin, dass sich das Signalisierungsprinzip bei Lernenden mit geringem Vorwissen besonders positiv auf das Lernen auswirkt. Stattdessen zeigt die Analyse von Schneider et al. (2018) einen positiven Effekt hohen Vorwissens auf die Wirkung des Signalisierungsprinzips. Auch Richter und Scheiter (2019) untersuchten die Rolle des Vorwissens auf die Wirkung des Signalisierungsprinzips. Ihre Ergebnisse zeigen Auswirkungen eines Interaktionseffekts von Vorwissen und Signalisierungsprinzip auf die Informationssuche 
beim Lernen mit Texten. Ein niedriges Vorwissen führt in Kombination mit den signalisierenden Hinweisen dazu, dass Textinformationen später erfasst werden. Dies ist bemerkenswert, da verschiedene Studien zum Signalisierungsprinzip und zum Split-Attention-Effekt eine Art Textdominanz zeigen (siehe Hegarty und Just 1993; Ozcelik et al. 2010; Schmidt-Weigand et al. 2010). So untersuchten Hegarty und Just (1993) das Blickverhalten während des Lernens von visuellen Darstellungen mit ergänzenden Texterläuterungen. Ihre Ergebnisse zeigen, dass Texte tendenziell vor den visuellen Darstellungen betrachtet und verarbeitet werden. Hegarty und Just (1993) schlossen daraus, dass beim Lernen den Texten besondere Bedeutung beigemessen wird. Bestätigung finden diese Ergebnisse durch die Studie zum Modalitäts- und Kontiguitätseffekt von Schmidt-Weigand et al. (2010), die ebenfalls Split-AttentionEffekte und speziell die Textbezogenheit bei Lernmaterialien nachweisen.

Neben dem Vorwissen scheint auch der Inhaltsgehalt von Hinweisen die Wirkung auf das Lernen zu beeinflussen. Hinweise mit geringem Inhaltsgehalt sind z. B. Spotlights oder Pfeile. Sie bieten keine inhaltliche Unterstützung für die kognitive Verarbeitung des Gesehenen an (vgl. de Koning und Jarodzka 2017). So gelingt zwar das Lenken der visuellen Aufmerksamkeit gut mittels Spotlights (de Koning et al. 2010; Jarodzka et al. 2013), Farbänderungen (z. B. Ozcelik et al. 2010) und Pfeilen (Kriz und Hegarty 2007; Boucheix und Lowe 2010). Zum besseren Verständnis tragen aber diese Hinweisformen nicht immer bei (Kriz und Hegarty 2007; Boucheix und Lowe 2010; de Koning et al. 2010). Dennoch haben rein aufmerksamkeitssteuernde Hinweise ihre Berechtigung in Lernmaterialien. Sie können z. B. die Informationssuche verkürzen (Spotlights, Jarodzka et al. 2013 und Farbänderungen, Ozcelik et al. 2010), das Wiedergeben von Gesehenem verbessern (Spotlights, de Koning et al. 2007) und das Erinnern an den Ort des Gesehenen unterstützen (Zoomen, Glaser und Schwan 2020).

Neben den aufmerksamkeitssteuernden Hinweisen gibt es auch Hinweisformen mit hohem Inhaltsgehalt und entsprechend stärkeren Anreizen, das Gesehene kognitiv zu verarbeiten. Beispiele sind verbale Erläuterungen in Form gesprochener Texte bzw. geschriebener Textfelder. Bei einem überwiegend bildbasierten Lernmaterial haben vor allem gesprochene Texte positive Effekte auf die visuelle Aufmerksamkeit und den Lernerfolg (z. B. Glaser und Schwan 2015). Eine Erklärung für die bessere Eignung gesprochener Texte im Vergleich zu geschriebenen gibt die CTML (vgl. 2.2). Aufgrund der begrenzten Kapazität der Verarbeitungskanäle, ist es demnach sinnvoll zusätzliche Ressourcen des auditiven Verarbeitungskanals zu nutzen und so dem Modalitätsprinzip (vgl. 2.2.1) zu folgen.

Eine weitere Möglichkeit auf den fehlenden Inhaltsgehalt von rein aufmerksamkeitssteuernden Hinweisen zu reagieren ist, sie mit inhaltlichen Hinweisen zu kombinieren. Scheiter und Eitel (2015) zeigen, dass eine Kombination aus farblichen Hervorhebungen und kursiven Schriftweisen von Begriffen, Pfeilen sowie Textfeldern die Informationssuche verkürzen, die visuelle Aufmerksamkeit auf Informationen erhöht und den Lernerfolg verbessert. Sie weisen ferner in einer Mediatoranalyse nach, dass die visuelle Aufmerksamkeit die Varianzen im Lernerfolg komplett erklärt (vgl. 2.5).

Auch die Ergebnisse von Xie et al. (2019) sowie Glaser und Schwan (2020) zeigen jeweils, dass Kombinationen aus visuellen und auditiven Hinweisen den Lernerfolg 
erhöhen können. Speziell die Ergebnisse aus Korrelationsanalysen von Xie et al. (2019) zeigen Verknüpfungen zwischen Maßen der visuellen Aufmerksamkeit und dem Lernerfolg. Zum einen weisen sie einen Zusammenhang moderater Effektstärke zwischen der Informationssuche und der Lernleistung nach. Eine kürzere Suchzeit nach relevanten Informationen ist hier verknüpft mit einem besseren Lernergebnis. Zum anderen zeigen sie, dass zwischen mittlerer Blickdauer und Lernleistung ein positiver Zusammenhang von kleiner bis mittlerer Effektstärke besteht. Eine längere Blickdauer auf relevante Informationen fällt mit besseren Lernergebnissen zusammen.

Zusammengefasst zeigen die Befunde, dass vor allem visuelle Hinweise wie Pfeile, Spotlights und Markierungen i.d.R. über die reine Aufmerksamkeitssteuerung hinausgehende Maßnahmen zur kognitiven Verarbeitung der Informationen benötigen, um bezüglich des Lernerfolgs wirksam zu werden (de Koning und Jarodzka 2017). Dies kann wie Xie et al. (2019) sowie Glaser und Schwan (2015, 2020) zeigen, durch Kombinieren von Hinweisen oder durch das Implementieren inhaltsbezogener Informationen in visuell/geschriebener oder auditiv/gesprochener Form erfolgen.

\subsection{Bedeutung der Befunde für Hinweise in Demonstrationsvideos}

Demonstrationsvideos können komplexe Handlungsschritte und gleichzeitig auftretende Änderungen zeigen. In solchen Fällen sind speziell für die Lokalisierung einzelner Elemente im Lernmaterial (vgl. Glaser und Schwan 2020) oder die Reihenfolge von Handlungsschritten (vgl. de Koning et al. 2010) aufmerksamkeitssteuernde visuelle Hinweise besser geeignet als verbale. Die visuellen Hinweise wie etwa Spotlights können unverwechselbar und direkt auf den relevanten Bereich im Video hinweisen. Für sich allein genommen, sind aber rein visuelle Hinweise wie Spotlights für das Lernen vermutlich ineffektiv, da sie nur die visuelle Aufmerksamkeit steuern (vgl. 2.2.2 und de Koning und Jarodzka 2017). Daher sollten sie mit inhaltsorientierten Hinweisformen kombiniert werden. Eine Möglichkeit ist das Anbieten von Textfeldern (vgl. Scheiter und Eitel 2015) und/oder Labeln (vgl. Ozcelik et al. 2010). Geschriebene Texte bieten Informationen in vorgegebener Reihenfolge nacheinander an. Die Informationen sind zwar erst nach vollständigem Lesen verständlich, können dafür aber im eignen Lerntempo verarbeitet und bei Bedarf mehrfach gelesen werden. Jedoch sind bei Kombinationen aus geschriebenen Texten und visuellen Hinweisen Split-Attention-Effekte zu befürchten, da die Aufmerksamkeit zwischen visuellen Hinweisen und den dadurch markierten Bereichen aufgeteilt werden muss. Eine Alternative ist das Geben von auditiven Hinweisen. Hier werden Split-Attention-Effekte weitestgehend vermieden. Allerdings könnten auditive Hinweise wie etwa ,links neben der Spannungsquelle“ zu einer kognitiv belastenden Suche führen (vgl. Glaser und Schwan 2020), insbesondere wenn wegen mangelndem Vorwissen die Gerätschaften und Fachtermine unbekannt sind. Hinzu kommt, dass bei auditiven Hinweisen der Hörer der Reihenfolge und dem Tempo des Sprechers folgen muss.

Die Zusammenstellung zeigt Vor- und Nachteile visueller bzw. auditiver Hinweise. In der Regel überwiegen für die meisten Lernziele und kurze, unkomplizierte 
Texte die Vorteile auditiver Hinweise in Lernmaterialien hinsichtlich des Lernerfolgs und der Modalitätseffekt kommt zum Tragen. Im Fall von physikalischen Fachbegriffen und Beschreibungen kann aber eine gewisse Komplexität angenommen werden, so dass der Modalitätseffekt aufgrund der Transienz eingeschränkt sein könnte (vgl. 2.2.1).

\subsection{Visuelle Aufmerksamkeit und deren Beeinflussung durch Hinweise}

Bei der Verarbeitung von Informationen in Lernmedien ist deren visuelle Wahrnehmung eine zentrale Voraussetzung für das Lernen (Just und Carpenter 1976). Aus verschiedenen Eye-Tracker-Metriken lassen sich Rückschlüsse auf die visuelle Aufmerksamkeit ziehen.

- Die Zeit vom Erscheinen bis zur ersten Fixation gibt Auskunft über die Geschwindigkeit der visuellen Informationssuche (Jarodzka et al. 2013) und kann dem kognitiven Prozess des Selektierens aus der CTML (vgl. 2.2) zugeordnet werden (Alemdag und Cagiltay 2018). Ergebnisse von Eye-Tracker-Studien zeigen, dass das Geben von Hinweisen die Informationssuche verkürzt (z. B. Ozcelik et al. 2010; Jarodzka et al. 2013; Scheiter und Eitel 2015). Xie et al. (2019) zeigen, dass für die Informationssuche visuell-auditive oder rein visuelle Hinweise besser geeignet sind als rein auditive.

- Die Anzahl der Fixationen ist ein Maß, das darauf hindeutet, wie intensiv ein Betrachter zwischen fixierten Informationen Verknüpfungen zieht (z. B. Scheiter und Eitel 2015; Alemdag und Cagiltay 2018). Aus Eye-Tracker-Studien zum Signalisierungsprinzip geht hervor, dass durch das Geben von Hinweisen die Fixationsanzahl gesteigert werden kann (Ozcelik et al. 2010; Boucheix und Lowe 2010; Scheiter und Eitel 2015). Xie et al. (2019) zeigen, dass visuell-auditive oder rein visuelle Hinweise zu höheren Fixationsanzahlen auf die adressierten Informationen im Lernmaterial führen als rein auditive Hinweise.

- Die mittlere Blickdauer gibt die Zeitdauer für das durchschnittliche Betrachten eines festgelegten Informationsbereiches (AOI) an. Die Zahl der Fixationen und die mittlere Blickdauer können dem kognitiven Prozess des Organisierens der CTML (vgl. 2.2) zugeordnet werden (Alemdag und Cagiltay 2018) und auf den Grad der mentalen Anstrengung hinweisen (vgl. Jarodzka et al. 2015). Ozcelik et al. (2010) interpretieren eine lange mittlere Blickdauer mit eher anspruchsvollen Aufgaben und entsprechend höherer mentaler Anstrengung. Hinweise führen dazu, dass die durch die Hinweise adressierten Informationen im Lernmaterial länger betrachtet werden als in Lernmaterialien ohne Hinweise (Boucheix und Lowe 2010; Ozcelik et al. 2010; Glaser und Schwan 2015; Xie et al. 2019). Schmidt-Weigand et al. (2010) und Xie et al. (2019) zeigen, dass auch die Hinweismodalität die Blickdauer beeinflusst. Rein visuelle Hinweise ohne Inhaltsgehalt (z. B. Pfeile, Farben, Kreise) führen bei Xie et al. (2019) zur längsten Blickdauer, gefolgt von kombinierten visuell-auditiven Hinweisen, rein auditiven Hinweisen und keinen Hinweisen. Jedoch führen visuelle Hinweise mit höherem Inhaltsgehalt wie geschriebene Texte im Vergleich zu entsprechenden auditiven Hinweisen zu kürzeren Blickdau- 
ern auf die durch die Texte adressierten Informationen (Schmidt-Weigand et al. 2010).

Diese Interpretationen der Eye-Tracker-Metriken werden in der vorliegenden Studie für die Messung der visuellen Aufmerksamkeit und Analyse der aufmerksamkeitssteuernden Wirkungen der gegebenen Hinweise genutzt.

\subsection{Wirkung der visuellen Aufmerksamkeit als Mediator}

Scheiter und Eitel (2015) als auch Xie et al. (2019) untersuchten die Rolle der visuellen Aufmerksamkeit als Mediator auf den Zusammenhang zwischen Hinweisen und Lernerfolg. Dabei war die visuelle Aufmerksamkeit durch Eye-TrackerMetriken bestimmt. Bei Scheiter und Eitel (2015) waren es die Zeit bis zur ersten Fixation und die Fixationsanzahl, die als Maße für die visuelle Aufmerksamkeit als Mediatoren in das Modell aufgenommen wurden. Für eine von drei abhängigen Variablen zeigen sich indirekte Effekte der Hinweise über die Mediatoren. Ein etwas anderes Bild zeigen die Analysen von Xie et al. (2019), bei denen die Zeit bis zur ersten Fixation, die Fixationsdauer und die Fixationsanzahl als Mediatoren in die Analysen eingingen. Die Ergebnisse zeigen keinen Mediationseffekt der visuellen Aufmerksamkeit auf den Zusammenhang zwischen Hinweisen und Lernerfolg.

\section{Forschungsfrage und Hypothesen}

Wie aus den Befunden zum Signalisierungsprinzip unter Beachtung der visuellen Aufmerksamkeit (vgl. 2.4 und 2.5) hervorgeht, ist noch nicht abschließend geklärt, welche Rolle die visuelle Aufmerksamkeit bei der Wirkung von Hinweisen auf das Lernen einnimmt. An diesem Punkt knüpft die vorliegende Studie an, indem sie versucht, einen möglichen Mediationsmechanismus der Hinweise über die visuelle Aufmerksamkeit auf den Lernerfolg aufzudecken. Die untersuchte Forschungsfrage lautet daher:

Vermittelt die visuelle Aufmerksamkeit der Lernenden die Wirkung der Hinweismodalität auf den Lernerfolg?

Es wird vermutet, dass die Hinweismodalität den Lernerfolg indirekt über die visuelle Aufmerksamkeit beeinflusst. Dabei sollte sich bezüglich des Lernerfolges ein totaler Effekt der Hinweismodalität auf den Lernerfolg einstellen, der auf dem Modalitätseffekt beruht. Eine Gruppe mit gesprochenen Hinweisen in Experimentiervideos sollte daher einen höheren Lernerfolg erzielen als eine Gruppe mit geschriebenen Hinweisen. Der direkte Effekt der Hinweismodalität sollte durch die Hinzunahme der visuellen Aufmerksamkeit als Mediator verschwinden. Speziell beim Erlernen der Fachsprache sollte die Transienz gesprochener Hinweise den Modalitätseffekt einschränken. 


\section{Methodik}

\subsection{Stichprobe und Design}

An der Studie nahmen 40 Lehramtsstudierende der Physik des 5. Fachsemesters teil. Das Durchschnittsalter der Probanden lag zwischen 21 und 25 Jahren (MW=22,72 Jahre, SD=2,50 Jahre). Die Teilnehmenden wurden zufällig zwei Versuchsbedingungen (Videos mit visuellen Hinweisen vs. Videos mit auditiven Hinweisen) zugeteilt. Dazu wurden nach der Akquise der 40 Probanden 20 Kärtchen für die visuelle bzw. 20 Kärtchen für die auditive Versuchsbedingung in eine Schuhschachtel gegeben. Die Kärtchen wurden von den Probanden nacheinander und ohne Ersetzung gezogen.

Als experimentelles Design wurde ein 2-Gruppen-Design mit der unabhängigen Variablen Hinweismodalität (auditiv vs. visuell) gewählt. Außerdem fand die Untersuchung einzeln unter Laborbedingungen statt. Eine Kontrollgruppe, in der die Videos ohne Hinweise zum Einsatz kamen, wurde nicht erhoben. Die zufällige Zuteilung und die Durchführung als Laborstudie sollten sicherstellen, dass sich mögliche konfundierende Variablen in beiden Versuchsbedingungen in gleichem Maße auswirken und folglich relative Vergleiche zwischen den Bedingungen möglich sind.

Der Studie lag ein Pre-Post-Design zugrunde. Nach dem Pre-Test, erfolgte die Einstellung und Kalibrierung des Eye-Trackers. Anschließend wurde das Video zur Wirbelstromscheibenbremse am Computer betrachtet, während der Eye-Tracker die Blickbewegungen aufzeichnete. Danach wurde der Eye-Tracker erneut kalibriert und das Video zum Waltenhofen Pendel angesehen. Auch hier zeichnete der Eye-Tracker die Blickbewegungen während dem Ablaufen des Videos auf. Den Abschluss der Studie bildete der Post-Test.

\subsection{Lernmaterial}

Für die Studie wurden zwei Standardexperimente zur Demonstration von Wirbelströmen gefilmt (Scheibenbremse und Waltenhofen Pendel) und visuelle bzw. auditive Hinweise implementiert. Die Teilnehmerinnen und Teilnehmer sollten nach der Betrachtung der Videos den Aufbau der gezeigten Experimente korrekt skizzieren und die Handlungsschritte der Durchführung korrekt beschreiben können. Im Mittelpunkt stand somit der Erwerb deklarativen Wissens über klassische Schulexperimente, das häufig in Staatsexamina abgefragt wird und zudem für das spätere Berufsleben der Teilnehmerinnen und Teilnehmer relevant ist.

\subsubsection{Demonstrationsvideos zu Wirbelströmen}

Beide Videos zeigten zunächst in der ersten Phase das Experimentiermaterial und gaben als Hinweise jeweils die Bezeichnung der verwendeten Materialien an (z. B. „Spannungsquelle“ siehe Abb. 1). Anschließend präsentierten die Videos jeweils den Aufbau. Die Hinweise zum Aufbau betonten besondere Spezifikationen wie bspw. „Das Pendel kann durch den Spalt des Elektromagneten schwingen.“ oder „Der Elektromagnet wird mit Gleichstrom betrieben.“. Schließlich zeigten die Videos 
Abb. 1 Video zum Waltenhofen Pendel mit visuellen Hinweisen (Textfeld und Spotlight)

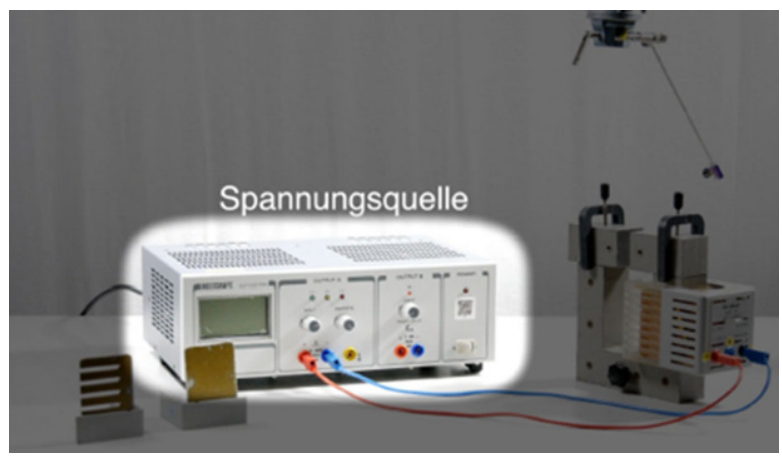

in der dritten Phase zwei Mal die Durchführung der Experimente. Während der ersten Durchführung wurden jeweils Hinweise gegeben, die sich auf die Variation der unabhängigen Variablen bezogen (z. B. ,Elektromagnet an“ bzw. „Elektromagnet aus“). In der zweiten Durchführung der Experimente wurden in beiden Videos keine Hinweise mehr eingeblendet.

\subsubsection{Textfelder und Spotlights vs. gesprochene Hinweise in Videos}

Die Hinweise in den beiden Videos wurden bezüglich ihrer Modalität variiert (visuell vs. auditiv). In der visuellen Versuchsbedingung erschienen sie als Textfelder und Spotlights, welche die jeweils passenden Bereiche im Video hell hervorhoben (siehe Abb. 1). Textfelder und Spotlights waren räumlich nahe beisammen und gleich lange eingeblendet (räumliches und zeitliches Kontiguitätsprinzip).

In der auditiven Versuchsbedingung wurden die gleichen Sachinformationen wie in der visuellen Bedingung angeboten allerdings nicht in Form von Textfeldern, sondern gesprochen. Weiterhin wurden als Ersatz für die visuellen Spotlights aufmerksamkeitssteuernde Anweisungen wie „links im Vordergrund“ gesprochen angeboten (siehe Abb. 2).

Fest vorgegebene Präsentationszeiten stellten eine konstante Lernzeit zwischen den Versuchsbedingungen sicher. Dazu wurden die Präsentationszeiten in beiden Versuchsbedingungen parallelisiert, indem die visuellen Hinweise exakt gleich lange erschienen wie die entsprechenden auditiven Hinweise.

\subsection{Instrumente}

\subsubsection{Eye-Tracker}

Mit dem EyeFollower von LC Technology (Abtastrate: $120 \mathrm{~Hz}$ bzw. alle 8,3 ms) und einem 24" Bildschirmmonitor mit der Auflösung 1920×1200 Pixel erfolgte die Messung der Eye-Tracker-Metriken für die Bestimmung der visuellen Aufmerksamkeit. Die Kalibrierung erfolgte durch 5 Punkte, wobei die durchschnittliche Differenz zwischen der tatsächlichen Punktposition und der gemessenen Blickposition kleiner als $0,4^{\circ}$ war. 
Abb. 2 Video zum Waltenhofen Pendel mit auditiven Hinweisen (gesprochener Text: ,Links hinter der geschlitzten Platte und der Platte ist eine Spannungsquelle zu sehen")

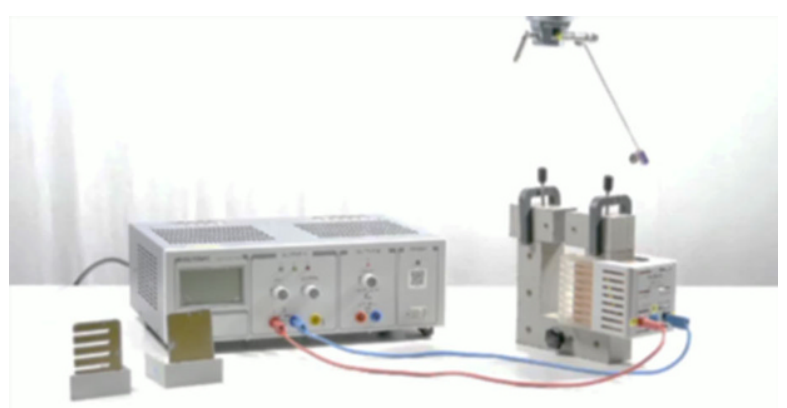

\subsubsection{Kontrollvariablen (Pre-test)}

Der schriftliche Pre-Test erfasste demografische Daten und Kontrollvariablen (siehe Anlage 2). Alle Skalen, außer die zum Vorwissen, waren Likert-Skalen mit fünf oder sechs Ausprägungen. Das Vorwissen wurde mittels vier Multipel-Choice-Items erfasst. Beispielitems und Testgütekriterien können Anlage 2 entnommen werden.

\subsubsection{Lernerfolg (Post-Test)}

Der schriftliche Post-Test prüfte die Lernergebnisse durch offene Fragen ab. Erfasst wurden die folgenden Fähigkeiten: (a) Angabe der Geräte, (b) fachsprachlich korrekte Bezeichnung der Geräte, (c) beschriftete Skizze und verbale Beschreibung des Aufbaus und (d) Beschreibung der Durchführung. Das offene Antwortformat sollte sicherstellen, dass sich der Fragebogen nicht auf die Beantwortung der Fragen auswirkt. Außerdem wurde erwartet, dass die Antworten zumindest teilweise Skizzen enthalten würden, die mit offenen Fragen besser erfasst werden können. Ein weiterer Grund war, dass mit offenen Fragen ohne vorgebende Einschränkung das Wissen erfasst werden kann. Weitergehende Fragen zu den Experimenten, wie die Diskussion von Versuchsergebnissen oder von Messgenauigkeiten etc. sind zwar Teil der fachdidaktischen Ausbildung, lassen sich aber besser direkt am Realexperiment behandeln. Daher beschränkt sich die Wissenserfassung auf die in den Videos vermittelten Inhalte.

Bei den Likert-Skalen wurden Mittelwerte berechnet. Bei den offenen Fragen wurde der Summenscore gebildet und dieser ins Verhältnis zum Gesamtscore gesetzt. Zudem wurde für die Skalenbildung der offenen Fragen ein Kategorienschema festgelegt, das der Anlage 1 entnommen werden kann.

Zur Prüfung der Objektivität der Kategorienzuordnung kodierte eine unabhängige Raterin 3/4 der Antworten erneut. Als Übereinstimmungsmaß wurde für den CohensKappa-Koeffizienten der Wert $\kappa=0,71$ ermittelt. Dieser Wert spricht laut Bortz und Döring (2006) für eine gute Übereinstimmung der beiden Rater, so dass die Daten der Skala als hinreichend objektiv angesehen werden können.

Zur Prüfung der inhaltlichen Validität schätzten drei Lehrkräfte die offenen Fragen zum Lernergebnis und das Kategorienschema aus Anlage 1 hinsichtlich der Eignung für das Messen deklarativen Wissens zu den gezeigten Experimenten. Sie hielten 
einstimmig die Fragen für sehr gut geeignet. Das Kategorienschema schätzte eine Lehrkraft als gut geeignet und zwei schätzten es als sehr gut geeignet ein.

Die Schätzung der internen Konsistenz erfolgte durch die Berechnung von Cronbachs Alpha. In die Analyse gingen die oben genannten vier Items ein. Der ermittelte Wert für Cronbachs Alpha lag bei 0,809, was auf eine relativ gute Passung der Items in der Skala hindeutet. Zwar ließe sich die interne Konsistenz durch Entfernen eines Items etwas verbessern, allerdings würde dies auf Kosten der inhaltlichen Validität gehen.

\subsection{Auswertung}

Um die Forschungsfrage zu beantworten, wie die Hinweismodalität ihre Wirkung auf den Lernerfolg ausübt und inwieweit die visuelle Aufmerksamkeit dabei als vermittelnder Prozess beteiligt ist wurden mit dem SPSS-Makro PROCESS v3.4 von Hayes Mediationsanalysen berechnet. Als unabhängige Variable ging die Hinweismodalität ein. Als Mediatoren ging die visuelle Aufmerksamkeit ein. Die abhängige Variable war zunächst der kumulierte Lernerfolg und anschließend die fachsprachliche Fähigkeit (vgl. Anlage 1).

Für das Signifikanzlevel von 5\% wurden die entsprechenden 95\%-Konfidenzintervalle berechnet. Das Bootstrap-Verfahren war bias-corrected und die Anzahl der Bootstrap Stichproben betrug 10.000 .

\section{Ergebnisse}

Mittelwerte und Standardabweichungen können Anlage 3 entnommen werden. Die Teststärke wurde im Nachhinein mittels G*Power 3.1 bestimmt. Die Effektstärke $f^{2}$ wurde über die Änderungen in den $R^{2}$-Werten der Prädiktoren (Blickdauer, Blickhäufigkeit und Hinweismodalität) in $\mathrm{G}^{*}$ Power berechnet und lag für die Blickdauer bei $f^{2}=2,79$, für die Blickhäufigkeit bei $f^{2}=0,63$ und für die Hinweismodalität bei $f^{2}=0,30$. Für das Signifikanzlevel von 5\%, 40 Probanden und die kleinste Effektstärke $f^{2}=0,30$ ergab die Analyse eine Power 0,92.

Das verwendete SPSS-Makro PROCESS v3.4 von Hayes basiert auf linearen Regressionen. Daher gelten laut Hayes (2018) ähnliche Voraussetzungen, die geprüft werden sollten (siehe Anlage 4).

\subsection{Kumulierter Lernerfolg}

Nachfolgend wird mittels Mediationsanalyse untersucht, ob die visuelle Aufmerksamkeit den Effekt der Hinweismodalität auf den Lernerfolg mediiert (siehe Abb. 3). Wenn wie erwartet, die Hinweismodalität Änderungen in der visuellen Aufmerksamkeit bewirkt und diese ihrerseits Änderungen im Lernerfolg zur Folge hat, dann sollte der direkte Effekt der Hinweismodalität auf den Lernerfolg vollständig durch den indirekten Effekt mediiert werden und somit „verschwinden“.

Zunächst zeigen die Ergebnisse einen totalen Effekt der Hinweismodalität auf den Lernerfolg $(B=18,10, p<0,001,95 \% C I=[12,33,23,88])$. Der partiell standa- 


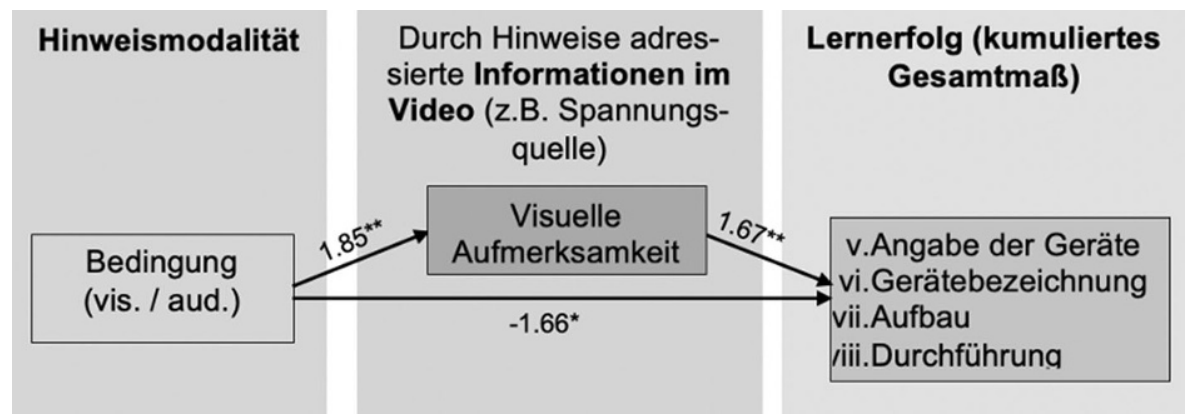

Abb. 3 Mediationsmodell mit einem Mediator (visuelle Aufmerksamkeit, gemessen über die Blickhäufigkeit), der sich kausal lokalisiert zwischen der unabhängigen Variable Hinweismodalität (Bedingung: visuell $=0$, auditiv $=1$ ) und der ab. Variable Lernerfolg befindet. Die angegebenen Werte der $\beta$-Gewichte sind standardisiert. $\left(* * p<0,001, * p<0,05, R^{2}=0,879\right)$

risierte totale Effekt beträgt 1,43. Wenn eine Person statt in der visuellen Bedingung in der auditiven Bedingung ist, dann steigert sich ihr Lernerfolg um 1,43 Standardabweichungen.

Nimmt man den Mediator visuelle Aufmerksamkeit in das Modell auf, wird dieser von der Hinweismodalität signifikant vorhergesagt $(B=22,78, b=1,85, p<0,001$, $95 \% C I=[19,93,25,63])$. Die visuelle Aufmerksamkeit sagt wiederum den Lernerfolg signifikant vorher $(B=1,71, b=1,67, p<0,001,95 \% C I=[1,27,2,16])$. Der partiell standardisierte indirekte Effekt beträgt für den Pfad über die visuelle Aufmerksamkeit 3,09. Ist eine Person statt in der visuellen Bedingung in der auditiven Bedingung, dann steigert sich ihr Lernerfolg über die visuelle Aufmerksamkeit um 3,09 Standardabweichungen.

Weiterhin zeigen die Ergebnisse, dass der direkte Effekt der Hinweismodalität auf den Lernerfolg nicht verschwindet. Im Vergleich zum totalen Effekt ohne Mediator kehrt sich der direkte Effekt durch das Hinzunehmen der visuellen Aufmerksamkeit als Mediator um $(B=-20,94, p=0,001,95 \% C I=[-32,88,-9,00])$. Der partiell standarisierte direkte Effekt beträgt $-1,66$. Wenn eine Person statt in der visuellen Bedingung in der auditiven Bedingung ist, schwächt der direkte Effekt den Lernerfolg um 1,66 Standardabweichungen.

Einen differenzierteren Blick auf die Wirkung der Hinweismodalität erlauben separate Analysen der Unterkategorien des Lernerfolgs. Dabei ist es laut Hayes (2018) unerheblich, ob die direkten und indirekten Auswirkungen der unabhängigen Variablen gleichzeitig in einem Modell für alle abhängige Variablen oder für jede abhängige Variable einzeln in mehreren separaten Modellen geschätzt werden (sofern die abhängige Variable nur durch die Mediatoren und die unabhängige Variable bestimmt wird).

\subsection{Fachsprachlich korrekte Bezeichnung der Geräte}

Mit einer weiteren Mediationsanalyse wird geprüft, ob die Hinweismodalität die Fähigkeit zur Anwendung der Fachsprache vorhersagt und ob der direkte Pfad durch 
die visuelle Aufmerksamkeit mediiert wird. Als Mediator gehen die visuelle Aufmerksamkeit bezogen auf Textfelder und jene bezogen auf das Experiment ein.

Ein totaler Effekt der Hinweismodalität auf die Fähigkeit zur Anwendung der Fachsprache liegt vor $(B=-2,80, p<0,001,95 \% C I=[-3,64,-1,96])$. Der partiell standarisierte totale Effekt beträgt $-2,14$. Wenn eine Person statt in der visuellen Bedingung in der auditiven Bedingung ist, dann schneidet sie bei der Verwendung der Fachsprache um 2,14 Standardabweichungen schlechter ab.

Wird die visuelle Aufmerksamkeit auf das Experiment und jene bezogen auf die Textfelder in das Modell aufgenommen, sagt die Hinweismodalität diese beiden Mediatoren signifikant vorher (visuelle Aufmerksamkeit bezüglich Textfelder: $B=-11,19, b=1,76, p<0,001,95 \% C I=[-13,11,-9,26]$, visuelle Aufmerksamkeit bezogen das Experiment: $B=22,78, b=1,85, p<0,001,95 \% C I=[19,93$, 25,63]), welche wiederum die Anwendung der Fachsprache signifikant vorhersagen (visuelle Aufmerksamkeit bezogen das Experiment: $B=0,13, b=1,16$, $p=0,001,95 \% C I=[0,61,1,99]$, visuelle Aufmerksamkeit bezüglich Textfelder $B=0,90, b=0,42, p=0,002,95 \% C I=[0,34,1,46])$. Der direkte Effekt verschwin$\operatorname{det}(p=0,959)$.

\section{Interpretation und Diskussion}

Primäres Forschungsanliegen der vorliegenden Studie war zu untersuchen, inwieweit die Wirkung der Hinweismodalität auf den Lernerfolg durch die visuelle Aufmerksamkeit mediiert wird und wie groß die jeweils ermittelten totalen, direkten und indirekten Effekte sind. Eine Stärke dieser Arbeit liegt in einer differenzierten Betrachtung des Lernerfolgs, bei der die Wirkung der Hinweise für verschiedene Wissensbereiche separat betrachtet wird. Gegenüber früheren Eye-Tracking-Arbeiten zur Wirkung des Signalisierungsprinzips ermöglicht diese Studie, an Lernzielen orientierte Empfehlungen für die Gestaltung von videobasierten Lernmaterialien mit implementierten Hinweisen aus den Ergebnissen abzuleiten.

Gemäß der aufgestellten Hypothese sollte der Einfluss der Hinweismodalität auf den Lernerfolg vollständig über die visuelle Aufmerksamkeit mediiert werden (vgl. Abschn. 3). Es kann festgehalten werden, dass der Zusammenhang zwischen der Hinweismodalität und dem Lernerfolg partiell, aber nicht komplett durch die visuelle Aufmerksamkeit auf das Experiment im Video mediiert wird. Daher wird die Hypothese zurückgewiesen.

Dennoch geben die Ergebnisse der Mediationsanalyse aufschlussreiche Einblicke in die Wirkung der Hinweismodalität. So zeigt das Modell, in das der kumulierte Lernerfolg (Gesamtmaß) eingeht, dass dieser beim Lernen mit auditiven Hinweisen durchschnittlich höher ausfällt als beim Lernen mit visuellen Hinweisen (totaler Effekt). Bemerkenswert ist hierbei, dass sowohl die Richtung des totalen Effekts als auch die Lenkung der visuellen Aufmerksamkeit beim Lernen mit den auditiven Hinweisen besser gelungen ist als beim Lernen mit den visuellen Hinweisen. Die Teilergebnisse bestätigen die Annahme aus Abschn. 3, wonach die auditiven Hinweise zu insgesamt besseren Lernergebnissen und höheren visuellen Aufmerksamkeit auf experimentelle Bildbereiche führen sollten. Das in der hier berichteten 
Studie beobachtete Ergebnis lässt schlussfolgern, dass die gesprochenen Hinweise im Zusammenspiel mit den visuellen Reizen des Videos zu einem Modalitätseffekt führten, was den höheren Lernerfolg erklären könnte (vgl. Ginns 2005). Danach greift beim Lernen mit auditiven Hinweisen in bildbasierten Lernmaterialien der Modalitätseffekt, weil sich die Belastung auf zwei verschiedene Arbeitsgedächtnissysteme verteilt. Die Erklärung greift also eine zusätzliche Ressource auf, die beim Lernen mit visuellen Hinweisen (geschriebene Texte) in bildbasierten Lernmaterialien ungenutzt bleibt. Rummer et al. (2008) kritisieren diese Annahme. Sie heben hervor, dass der Modalitätseffekt weniger von der Textmodalität als von den Anforderungen während des Verarbeitens von Texten und Bildern abhängt. Zudem könnten speziell in der auditiven Bedingung die sichtbaren Abläufe im Video wie eine Art visueller Hinweis gewirkt und folglich den Blick auf das Experiment gelenkt haben. Diese Vermutung wird u.a. durch empirische Befunde gestützt, die zeigen, dass Bilder generell eine aufmerksamkeitssteuernde Funktion haben (vgl. Peeck 1994; Brünken und Leutner 2001). Ein anderer Grund könnte darin liegen, dass die kognitive Verarbeitung der Textfelder, welche neben den Spotlights Teil der visuellen Hinweise waren, die Aufmerksamkeit mehr gebunden haben, als ursprünglich angenommen wurde. Texten werden generell beim Lernen eine besondere Bedeutung beigemessen. So zeigen Hegarty und Just (1993) eine Art Text-zuerstStrategie. Bestätigung finden diese These durch die Studie zum Modalitäts- und Kontiguitätseffekt von Schmidt-Weigand et al. (2010), die speziell die Textbezogenheit bei Lernmaterialien mit Kombinationen aus geschriebenen Texten und Visualisierungen nachweisen. Schmidt-Weigand et al. (2010) merken an, dass die Text-zuerstStrategie für statische Visualisierungen sinnvoll zu sein scheint, da das Lesen der Texte die nachfolgende Verarbeitung der Visualisierung erleichtert. Auf der anderen Seite könnte bei dynamischen und zeitlich nicht steuerbaren Visualisierungen das Vertrauen in Texte das Risiko erhöhen, eine Information zu verpassen. Übertragen auf die vorliegende Studie könnte demnach die Text-zuerst-Strategie dazu geführt haben, dass das Experiment entsprechend weniger häufig und lange betrachtet wurde und infolge dessen Informationen verpasst wurden. Von empirischen Studien abgesehen sprechen auch inhaltliche Argumente für eine Tendenz der Teilnehmenden zur Text-zuerst-Strategie. Die Textfelder enthielten u.a. die fachsprachlich korrekten Bezeichnungen der Geräte, welche die Betrachter vermutlich nicht in ihrem aktiven Wortschatz haben. Dem gegenüber stehen die durch Spotlights hervorgehobenen Geräte oder Versuchshandlungen. Sie könnten speziell in der Gruppe mit den visuellen Hinweisen den Eindruck hinterlassen haben, dass die Bildinformation einfacher sei als die Textinformation.

Außerdem zeigen die Mediationsergebnisse, dass sich der indirekte Effekt der Hinweismodalität über die visuelle Aufmerksamkeit positiv auf den Lernerfolg auswirkt. Je häufiger das Experiment im Video angesehen wird, desto größer fällt der Lernerfolg aus. Dieses Ergebnis deckt sich mit Befunden anderer Eye-Tracking-Studien, die positive Zusammenhänge zwischen Blickhäufigkeit und Lernerfolg zeigen (vgl. Alemdag und Cagiltay 2018) und legt die Vermutung nahe, dass die fokussierten Experimentierbereiche im Video intensiv verarbeitet wurden (vgl. Chen et al. 2014). Interessant ist hier, dass die indirekten Effekte durch einen entgegengesetzt gerichteten und schwächeren direkten Effekt der Hinweismodalität auf den Lerner- 
folg geschwächt werden. Dies lässt vermuten, dass weitere Faktoren die Wirkung der Hinweise auf das Lernen beeinflussen könnten. Beispielsweise könnte die kognitive Belastung von der Hinweismodalität beeinflusst sein und zudem den Lernerfolg vorhersagen. Denkbar ist, dass in der Bedingung mit visuellen Hinweisen aufgrund des Split-Attention-Effekts zwischen Textfeldern und Experiment die extrinsische kognitive Belastung erhöht ist. Sie resultiert nicht aus der Komplexität des Lerninhaltes, sondern aus der Gestaltung des Lernmaterials. Die extrinsische kognitive Belastung sollte so gering wie möglich ausfallen, da hier unnötig kognitive Ressourcen verschenkt werden. Die extrinsische kognitive Belastung kann sich auf die visuelle Aufmerksamkeit auswirken (Jarodzka et al. 2015) und durch das Binden von Ressourcen auch die Informationsverarbeitung beeinträchtigen (vgl. Sweller et al. 2011). Dies könnte die umgekehrte Richtung des direkten Effekts erklären. Dieser spräche nämlich dann für einen höheren Lernerfolg beim Lernen mit visuellen Hinweisen, wenn eine hohe visuelle Aufmerksamkeit auf das Experiment sichergestellt werden könnte.

Bemerkenswert ist auch das Ergebnis des zweiten Mediationsmodells, in das statt des gesamten Lernerfolgs nur die Fähigkeit die Fachsprache korrekt anzuwenden als abhängige Variable eingeht. Die Ergebnisse dieser Analyse deutet auf eine Stärke der Textfelder hin. Der totale Effekt zeigt, dass beim Lernen mit visuellen Hinweisen die Anwendung der Fachsprache besser unterstützt wird als beim Lernen mit auditiven Hinweisen. Dies verwundert nicht, da die Textfelder verglichen mit den auditiven Hinweisen den Vorteil haben, dass der Betrachter die unbekannten Fachbegriffe im eigenen Tempo lesen und verarbeiten kann. Im Gegensatz dazu muss der Hörer des gesprochenen Hinweises den Fachbegriff im Gedächtnis behalten. Außerdem lassen sich vermutlich in der visuellen Bedingung die Fachbegriffe in den Textfeldern und die zugehörigen Bauteile im Experiment leichter durch das Zusammenspiel zwischen Textfeld und Spotlight zuordnen als in der auditiven Bedingung. Diese Vermutungen werden durch die Ergebnisse von Glaser und Schwan (2020) unterstützt. Sie betonen, dass visuelle Hinweise das Gedächtnis weniger belasten als auditive und sich dies besonders auf das Erinnern an den Ort eines Elements in einem Bild auswirken kann. Im Gegensatz zum Modell, in das der Lernerfolg als Gesamtmaß einging, liegt hier eine vollständige Mediation vor. Die Aufnahme der visuellen Aufmerksamkeit auf das Experiment und jener auf die Textfelder als Mediatoren bewirkt, dass der direkte Effekt der Hinweismodalität auf die Anwendung der Fachsprache nicht signifikant ausfällt.

\section{Einschränkungen}

Eye-Tracker-Studien sind aufgrund der Einzelmessungen mit einem hohen Zeitaufwand und aufwendiger Auswertung verbunden. Dies spiegelt sich im Stichprobenumfang wider (hier: $n=40$ ). Für das Auffinden großer Unterschiede zwischen den beiden Versuchsbedingungen war diese Stichprobe ausreichend groß. Jedoch könnte es sein, dass kleine Unterschiede zwischen den beiden Bedingungen eventuell nicht erkannt wurden. Daher sollten die vorliegenden Ergebnisse zur Wirkung visueller 
bzw. auditiver Hinweise auf den Erwerb verschiedener Wissensfacetten an einer größeren Stichprobe repliziert werden.

Eine weitere Einschränkung betrifft das Design der Studie. Ohne Kontrollgruppe kann eine Konfundierung der Hinweiswirkung mit der Wirkung anderer auftretender Faktoren nicht restlos ausgeschlossen werden. Jedoch sollte die hier vorgenommene Randomisierung mögliche Fremdeinwirkungen gleichmäßig auf die Bedingungen verteilt haben. Daher können zwar keine absoluten, aber zumindest relative Vergleiche zwischen den beiden Bedingungen angestellt werden.

Weiterhin wurde die Studie als kontrollierte Laborstudie unter Verwendung eines Eye-Trackers durchgeführt. In anderen Lernumgebungen, z. B. in Seminarsitzungen oder Schulklassen, können weitere Einflüsse zum Tragen kommen, die sich auf die visuelle Aufmerksamkeit auswirken. Demzufolge ist in der Laborstudie die Vielfalt der Störfaktoren im Vergleich zu Alltagssituationen reduziert. Dies ist aber insofern gewünscht, als der Fokus dadurch stärker auf die Einflüsse der Intervention, der Materialien und der Fachinhalte gerichtet bleibt. Situationsabhängig sind aber dann ggf. weitere Einflüsse zu berücksichtigen.

Will man die Ergebnisse auf andere Populationen übertragen, ist genau zu prüfen, ob gleichwertige Voraussetzungen vorliegen. Die Studienpopulation war genau definiert und bestand aus Studierenden eines Fachsemesters des Studiengangs gymnasiales Lehramt Physik. Die Lernenden hatten also ein grundlegendes fachliches Vorwissen $\mathrm{zu}$ dem behandelten Themengebiet, allerdings noch keine tiefgehende Vorerfahrung mit dessen experimenteller Behandlung. Solche Voraussetzungen sind auch in anderen Populationen häufig gegeben. Somit können die Ergebnisse dieser Studie zur Weiterentwicklung der universitären Lehre in MINT-Fächern beitragen, da hier besonders häufig dynamische Visualisierungen eingesetzt werden. Als Anknüpfungspunkt kann darüber hinaus diese Studie mit ihren Ergebnissen genutzt werden, um weitere Untersuchungen insbesondere mit Schülerinnen und Schülern durchzuführen.

\section{Fazit und Implikation}

Mit der gebotenen Vorsicht lassen sich einige Konsequenzen für die Lehrerbildung, für weiterführende Studien und für die Entwicklung von multimedialen und dynamischen Lernmaterialien formulieren:

1. Die Ergebnisse zeigen, dass visuelle bzw. auditive Hinweise zur Förderung des Wissenserwerbs unterschiedliche Stärken aufweisen. Die Stärke von auditiven Hinweisen in Videos ist es, hohe durchschnittliche Fixationszahlen auszulösen, zugleich Split-Attention-Effekte zu vermeiden und zu besseren Lernergebnissen zu führen (abgesehen von dem Erwerb der Fachsprache). Jedoch kann nicht pauschal gesagt werden, dass akustische Hinweise grundsätzlich visuellen vorzuziehen sind. So eignen sich speziell visuelle Textfelder für die Vermittlung von Begriffen der Fachsprache.

2. Insgesamt sind die hier gefundenen Ergebnisse besonders für Lehrende in der universitären Physiklehrerausbildung interessant. Sie könnten die hier gewonnenen 
Erkenntnisse unmittelbar aufgreifen und ihre visuellen Darstellungen zielorientiert durch aufmerksamkeitssteuernde auditive oder visuelle Hinweise ergänzen, um den Studierenden das Rezipieren von Informationen zu erleichtern.

3. Prospektiv können die hier gefundenen Erkenntnisse auch von Entwicklern von Erklär-/Experimentiervideos, Animationen oder Simulationen genutzt werden, um ihre Lehr-Lernmaterialien auf den aufmerksamkeitssteuernden Einsatz von Hinweisen hin zu prüfen und gegebenenfalls anzupassen.

4. Die Ergebnisse könnten einen Anstoß für zukünftige Studien geben, speziell die Abhängigkeit der Hinweiswirkung von der Hinweismodalität für verschiedene Lernziele/Wissensfacetten zu untersuchen. 


\section{Anhang}

\section{Anlage 1}

Tab. 1 Kategorienschema

\begin{tabular}{|c|c|c|}
\hline $\begin{array}{l}\text { Kriterium der } \\
\text { Lernergebnisse }\end{array}$ & Antworten & Punkte \\
\hline $\begin{array}{l}\text { (a) Angabe der } \\
\text { Geräte (ohne } \\
\text { Berücksichti- } \\
\text { gung der } \\
\text { Fachsprache) }\end{array}$ & $\begin{array}{l}\text { Scheibenbremse: Netzgerät, Kabel, Eisenkern mit Spule, Polschuhe, } \\
\text { Stativ, Scheibe, Drehlager } \\
\text { Waltenhofen-Pendel: Netzgerät, Kabel, Eisenkern mit Spule, Polschu- } \\
\text { he, Stativ, Pendel, Pendelkörper }\end{array}$ & $\sum 14$ \\
\hline (b) Fachsprache & $\begin{array}{l}\text { Scheibenbremse: Gleichspannungsnetzgerät, Kabel, Elektromagnet, } \\
\text { Polschuhe, Stativ, Aluscheibe, Drehlager } \\
\text { Waltenhofen-Pendel: Gleichspannungsnetzgerät, Kabel, Elektroma- } \\
\text { gnet, Polschuhe, Stativ, Pendel, Messingplatte/Messingkamm }\end{array}$ & $\sum 14$ \\
\hline $\begin{array}{l}\text { (c) } \\
\text { Versuchsaufbau }\end{array}$ & $\begin{array}{l}\text { Scheibenbremse: Verbindung des Elektromagneten mit der Spannungs- } \\
\text { quelle, Elektromagnet bestehend aus Spule mit Eisenkern, Positionie- } \\
\text { rung der Polschuhe auf dem Eisenkern, Scheibe im Freiraum zwischen } \\
\text { den Polschuhen } \\
\text { Waltenhofen-Pendel: Verbindung des Elektromagneten mit der Span- } \\
\text { nungsquelle, Elektromagnet bestehend aus Spule mit Eisenkern, Po- } \\
\text { sitionierung der Polschuhe auf dem Eisenkern, Verbindung zwischen } \\
\text { Pendelkörper und Pendel, Pendelkörper im Freiraum zwischen den Pol- } \\
\text { schuhen }\end{array}$ & $\sum 9$ \\
\hline $\begin{array}{l}\text { (d) Versuchs- } \\
\text { durchführung }\end{array}$ & $\begin{array}{l}\text { Scheibenbremse: Scheibe andrehen, Elektromagnet anschalten, Elektro- } \\
\text { magnet ausschalten } \\
\text { Waltenhofen-Pendel: Messingplatte auslenken, bis zum Stillstand } \\
\text { schwingen lassen, Messingplatte auslenken, Elektromagnet anschal- } \\
\text { ten, Elektromagnet ausschalten, Messingplatte durch Messingkamm } \\
\text { ersetzen, Messingkamm auslenken, Elektromagnet anschalten, Elektro- } \\
\text { magnet ausschalten }\end{array}$ & $\sum 12$ \\
\hline
\end{tabular}




\section{Anlage 2}

Tab. 2 Beispielitems und Testgütekriterien

\begin{tabular}{|c|c|c|c|}
\hline Skala & Beispielitem & Cronbachs' $\alpha$ & $n$ \\
\hline Vorwissen $^{\mathrm{a}}$ & $\begin{array}{l}\text { „Ich kann den Aufbau der Wirbelstrombremse skiz- } \\
\text { zieren.“ }\end{array}$ & 0,71 & 4 \\
\hline $\begin{array}{l}\text { Sach- und } \\
\text { Fachinteresse }^{b}\end{array}$ & $\begin{array}{l}\text { „Freiwillig würde ich mich nie mit Physik beschäfti- } \\
\text { gen.“ }\end{array}$ & $0,87-0,88$ & 9 \\
\hline Motivation $^{\mathrm{c}}$ & $\begin{array}{l}\text { „Ich setze mich beim Lernen mit den Videos ein, } \\
\text { weil ich etwas dazulernen möchte.“ }\end{array}$ & $0,69-0,82$ & 13 \\
\hline $\begin{array}{l}\text { Anstrengungs- } \\
\text { bereitschaft }^{\mathrm{d}}\end{array}$ & $\begin{array}{l}\text { „Ich werde mich bemühen, die Inhalte der Untersu- } \\
\text { chung möglichst gut zu verstehen.“ }\end{array}$ & 0,86 & 6 \\
\hline Selbstkonzept $\mathrm{e}^{\mathrm{e}}$ & $\begin{array}{l}\text { „Ich traue mir zu, Bauteile/Geräte in physikalischen } \\
\text { Experimenten zu Wirbelströmen zu bestimmen.“ }\end{array}$ & 0,80 & 5 \\
\hline
\end{tabular}

$N=40$

aEigenkonstruktion

bVier Items zum Fachinteresse (vgl. Hoffmann et al. 1998), fünf Items zum Sachinteresse an Experimenten zu Wirbelströmen (Eigenentwicklung in Anlehnung an das Interessenkonstrukt von Krapp 1992)

${ }^{c}$ Vier Subskalen: intrinsisch, identifiziert, introjiziert, external (adaptiert, vgl. Thomas und Müller 2016)

${ }^{\mathrm{d}}$ Adaptiert, vgl. Beer und Benischek (2018)

eSubskala individuell (adaptiert von Schöne et al. 2012) 


\section{Anlage 3}

Tab. 3 Mittelwerte und Standardabweichungen der Fragebogenskalen und Eye-Trackermetriken

\begin{tabular}{|c|c|c|c|c|c|c|c|c|}
\hline \multirow[t]{3}{*}{$\overline{\text { Skala }}$} & \multicolumn{4}{|c|}{ Pre-Test } & \multicolumn{4}{|c|}{ Post-Test } \\
\hline & \multicolumn{2}{|c|}{$\begin{array}{l}\text { Visuelle Hin- } \\
\text { weise }\end{array}$} & \multicolumn{2}{|c|}{$\begin{array}{l}\text { Auditive Hin- } \\
\text { weise }\end{array}$} & \multicolumn{2}{|c|}{$\begin{array}{l}\text { Visuelle Hin- } \\
\text { weise }\end{array}$} & \multicolumn{2}{|c|}{$\begin{array}{l}\text { Auditive Hin- } \\
\text { weise }\end{array}$} \\
\hline & $M$ & $S D$ & $M$ & $S D$ & $M$ & $S D$ & $M$ & $S D$ \\
\hline Vorwissen & 3,30 & 1,17 & 3,33 & 1,12 & - & & & \\
\hline Sachinteresse & 3,03 & 0,90 & 2,98 & 0,96 & & & & \\
\hline Fachinteresse & 3,38 & 0,92 & 3,23 & 0,89 & & & & \\
\hline Motivation & 4,65 & 0,68 & 4,70 & 0,58 & & & & \\
\hline Anstrengungsbereitschaft & 4,70 & 1,23 & 4,81 & 1,18 & & & & \\
\hline Selbstkonzept & 2,82 & 0,91 & 2,92 & 0,97 & & & & \\
\hline Geräteangabe & - & & & & 8,45 & 1,40 & 11,70 & 1,38 \\
\hline Bezeichnung & & & & & 10,10 & 1,52 & 7,30 & 1,18 \\
\hline Aufbau & & & & & 5,65 & 1,50 & 6,95 & 1,36 \\
\hline Durchführung & & & & & 8,40 & 1,57 & 10,50 & 2,33 \\
\hline \multirow[t]{3}{*}{ Skala } & \multicolumn{4}{|c|}{ Visuelle Hinweise } & \multicolumn{4}{|c|}{ Auditive Hinweise } \\
\hline & $m H$ & & $o H$ & & $m H$ & & $o H$ & \\
\hline & $M$ & $S D$ & $M$ & $S D$ & $M$ & $S D$ & $M$ & $S D$ \\
\hline $\begin{array}{l}\text { Mittlere Anzahl der Fi- } \\
\text { xationen auf Textfelder }\end{array}$ & 4,97 & 5,66 & - & & - & & - & \\
\hline $\begin{array}{l}\text { Mittlere Anzahl der } \\
\text { Fixationen auf das Ex- } \\
\text { periment }\end{array}$ & 32,67 & 12,30 & 22,01 & 9,64 & 44,06 & 4,68 & 21,83 & 8,12 \\
\hline $\begin{array}{l}\text { Mittlere Blickdauer auf } \\
\text { Textfelder (s) }\end{array}$ & 13,65 & 10,93 & - & & - & & - & \\
\hline $\begin{array}{l}\text { Mittlere Blickdauer auf } \\
\text { das Experiment (s) }\end{array}$ & 14,61 & 3,21 & 8,83 & 4,21 & 28,07 & 4,57 & 9,64 & 4,50 \\
\hline $\begin{array}{l}\text { Zeit vom Erscheinen } \\
\text { eines Hinweises bis } \\
\text { zur ersten Fixation des } \\
\text { Textfeldes (s) }\end{array}$ & 0,45 & 0,18 & - & & - & & - & \\
\hline $\begin{array}{l}\text { Zeit vom Erscheinen } \\
\text { eines Hinweises bis } \\
\text { zur ersten Fixation des } \\
\text { Experiments (s) }\end{array}$ & 0,57 & 0,15 & - & & 0,45 & 0,14 & - & \\
\hline
\end{tabular}

$m H$ mit Hinweisen, $o H$ Wiederholung ohne Hinweise 


\section{Anlage 4}

\section{Prüfung der Voraussetzungen}

Das verwendete SPSS-Makro PROCESS v3.4 von Hayes basiert auf linearen Regressionen. Daher gelten laut Hayes (2018) ähnliche Voraussetzungen, die geprüft werden sollten. Dazu wurden in Streudiagrammen die Beziehungen zwischen den Eye-Tracking-Maßen und dem kumulierten Lernerfolg bzw. untergeordnete Kriterien des Lernerfolgs dargestellt. Das Streudiagramm in Abb. 4 zeigt den Zusammenhang zwischen der Blickhäufigkeit auf das Experiment und dem kumulierten Lernerfolg. Es liegt ein näherungsweise linearer, positiver Zusammenhang vor. Je häufiger relevante Bereiche betrachtet werden, desto besser fällt das Lernergebnis aus. Keine linearen Zusammenhänge sind in Diagrammen erkennbar, in denen die mittlere Blickdauer pro Fixation bzw. die Zeit vom Erscheinen/Erklingen eines Hinweises bis zur ersten Fixation und der Lernerfolg abgetragen sind. Daher wurden diese Metriken aus den weiteren Analysen ausgeschlossen und die Quantifizierung der visuellen Aufmerksamkeit erfolgte auf Grundlage der Blickhäufigkeit.

Die Normalverteilung der Residuen wurde über P-P-Plots geprüft und war abgesehen von akzeptablen Abweichungen von der Normalverteilung unauffällig. Das bei der Mediationsanalyse verwendete Bootstrappingverfahren ist laut Hayes (2018) robust, so dass sich nur große Abweichungen von Normalverteilungen auf die Statistik auswirken sollten. Die Unabhängigkeit der Residuen wurde über die DurbinWatson-Statistik ermittelt. Ihr Wert lag mit 2,154 nahe bei 2, wonach keine Autokorrelation der Residuen vorlag (Field 2013). Das Streudiagramm der Residuen

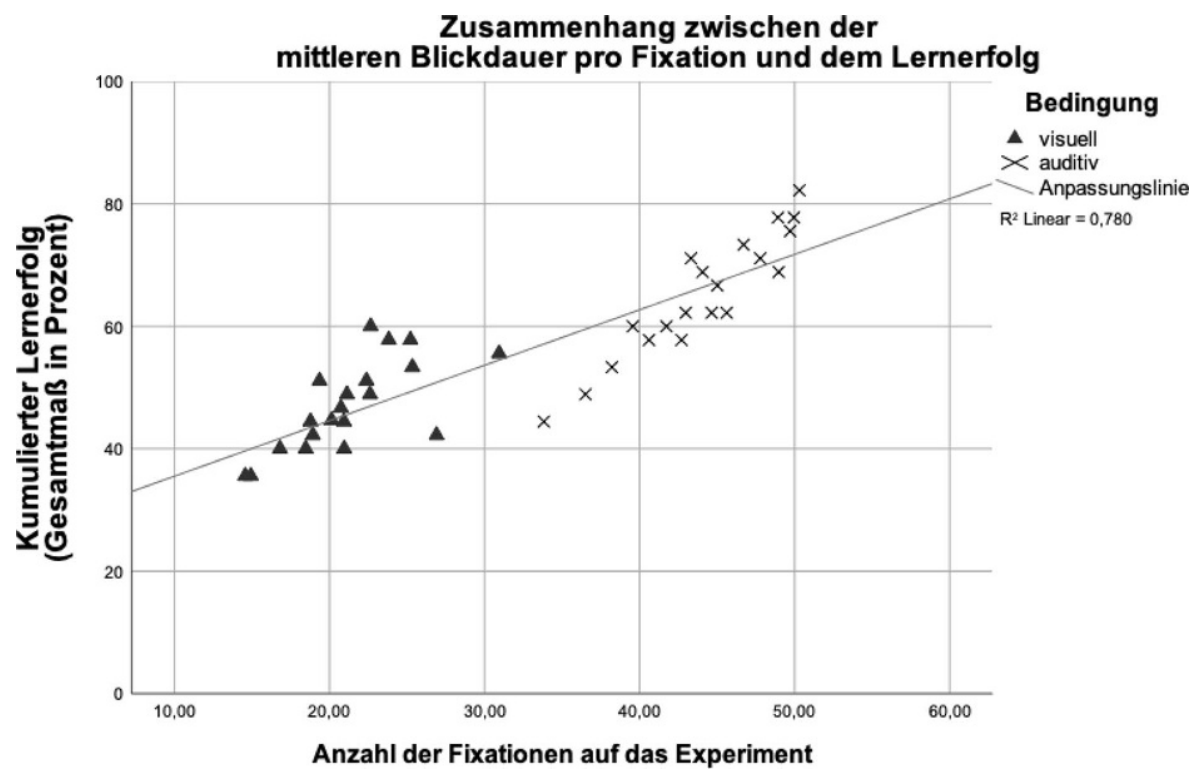

Abb. 4 Streudiagramm zum Zusammenhang zwischen der mittleren Fixationsanzahl und dem Lernerfolg $\left(R^{2}=0,780\right)$ 
(standardisierte Residuen gegen vorhergesagte Residuen) zeigte weder Trend noch Trichter. Folglich waren die Voraussetzung der Linearität und Homoskedastizität gegeben (Tabachnick und Fidell 2014). Der VIF-Wert der Kollinearitätsstatistik betrug für die die Blickhäufigkeit auf das Experiment unter 5 und für die Hinweismodalität 8,71. Letzterer war zwar erhöht, aber noch unter Grenze von 10. Die Analyse des Konditionsindexes ergab als höchsten Wert 20,70 und war somit deutlich kleiner als 30. Somit sollte keine Multikollinearität vorgelegen haben (vgl. Field 2013).

Weiterhin fällt auf, dass es auch Unterschiede zwischen den Versuchsbedingungen gibt. Die Gruppe mit den auditiven Hinweisen erzielt demnach größere Lernerfolge als die Gruppe mit den visuellen Hinweisen. Diese Unterschiede können in den folgenden Mediationsanalysen näher betrachtet werden.

Funding Open Access funding enabled and organized by Projekt DEAL.

Open Access Dieser Artikel wird unter der Creative Commons Namensnennung 4.0 International Lizenz veröffentlicht, welche die Nutzung, Vervielfältigung, Bearbeitung, Verbreitung und Wiedergabe in jeglichem Medium und Format erlaubt, sofern Sie den/die ursprünglichen Autor(en) und die Quelle ordnungsgemäß nennen, einen Link zur Creative Commons Lizenz beifügen und angeben, ob Änderungen vorgenommen wurden.

Die in diesem Artikel enthaltenen Bilder und sonstiges Drittmaterial unterliegen ebenfalls der genannten Creative Commons Lizenz, sofern sich aus der Abbildungslegende nichts anderes ergibt. Sofern das betreffende Material nicht unter der genannten Creative Commons Lizenz steht und die betreffende Handlung nicht nach gesetzlichen Vorschriften erlaubt ist, ist für die oben aufgeführten Weiterverwendungen des Materials die Einwilligung des jeweiligen Rechteinhabers einzuholen.

Weitere Details zur Lizenz entnehmen Sie bitte der Lizenzinformation auf http://creativecommons.org/ licenses/by/4.0/deed.de.

\section{Einhaltung ethischer Richtlinien}

Interessenkonflikt B. Watzka, C. Hoyer, B. Ertl und R. Girwidz geben an, dass kein Interessenkonflikt besteht.

Ethische Standards Alle durchgeführten Maßnahmen wurden in voller Übereinstimmung mit den berufsethischen Richtlinien der Deutschen Gesellschaft für Psychologie durchgeführt (DGPs, https://www.dgps. de/index.php?id=85). Zu Beginn der Studie wurde den Teilnehmerinnen und Teilnehmern mitgeteilt, dass die Daten dieser Studie nur zu Forschungszwecken verwendet werden und dass alle Daten anonym erfasst werden. Von allen Teilnehmerinnen und Teilnehmern liegt eine schriftliche Einverständniserklärung vor.

\section{Literatur}

Alemdag, E., \& Cagiltay, K. (2018). A systematic review of eye tracking research on multimedia learning. Computers \& Education, 125, 413-428.

Arnold, P., Kilian, L., Thillosen, A. M., \& Zimmer, G. M. (2013). Handbuch E-Learning. Lehren und Lernen mit digitalen Medien (3. Aufl.). Bielefeld: wbv.

Beer, R., \& Benischek, I. (2018). Leistungsfreude und Anstrengungsbereitschaft als Voraussetzung für erfolgreiches Lernen und Lehren, Open Online Journal for Research and Education, Tag der Forschung 2018. https://journal.ph-noe.ac.at/index.php/resource/article/view/463. Zugegriffen: 24. Okt. 2019.

Berney, S., \& Bétrancourt, M. (2016). Does animation enhance learning? A meta-analysis. Computers \& Education, 101, 150-167.

Bortz, J., \& Döring, N. (2006). Forschungsmethoden und Evaluation für Human- und Sozialwissenschaftler (4. Aufl.). Heidelberg: Springer. 
Boucheix, J.-M., \& Lowe, R. (2010). An eye tracking comparison of external pointing cues and internal continuous cues in learning with complex animations. Learning and Instruction, 20(2), 123-135.

Brünken, R., \& Leutner, D. (2001). Aufmerksamkeitsverteilung oder Aufmerksamkeitsfokussierung? Empirische Ergebnisse zur „Split-Attention-Hypothese“ beim Lernen mit Multimedia. Unterrichtswissenschaft, 29(4), 357-366.

Chen, S.-C., She, H.-C., Chuang, M.-H., Wu, J.-Y., Tsai, J.-L., \& Jung, T.-P. (2014). Eye movements predict students' computer-based assessment performance of physics concepts in different presentation modalities. Computers \& Education, 74, 61-72.

Field, A. P. (2013). Discovering statistics using IBM SPSS Statistics: And sex and drugs and rock 'n' roll (4. Aufl.). London: SAGE.

Gaudin, C. \& Chaliès, S. (2015). Video viewing in teacher education and professional development: A literature review. Educational Research Review, 16, 41-67.

Ginns, P. (2005). Meta-analysis of the modality effect. Learning and Instruction, 15(4), 313-331.

Glaser, M., \& Schwan, S. (2015). Explaining pictures: how verbal cues influence processing of pictorial learning material. Journal of Educational Psychology, 107(4), 1006-1018.

Glaser, M., \& Schwan, S. (2020). Combining verbal and visual cueing: fostering learning pictorial content by coordinating verbal explanations with different types of visual cueing. Instructional Science, 48 , $159-182$.

van Gog, T. (2014). The signaling (or cueing) principle in multimedia learning. In R. E. Mayer (Hrsg.), The Cambridge handbook of multimedia learning (2. Aufl. S. 263-278). New York: Cambridge University Press.

Hayes, A.F. (2018). Introduction to mediation, moderation, and conditional process analysis, a regressionbased approach (82. Aufl.). New York, London: Guilford.

Hegarty, M., \& Just, M. A. (1993). Constructing mental models of machines from text and diagrams. Journal of Memory and Language, 32(6), 717-742.

Höffler, T. N., \& Leutner, D. (2007). Instructional animation versus static pictures: a meta-analysis. Learning and Instruction, 17(6), 722-738.

Hoffmann, L., Häußler, P., \& Lehrke, M. (1998). Die IPN-Interessenstudie Physik. Kiel: IPN.

Jarodzka, H., van Gog, T., Dorr, M., Scheiter, K., \& Gerjets, P. (2013). Learning to see: Guiding students' attention via a Model's eye movements fosters learning. Learning and Instruction, 25, 62-70.

Jarodzka, H., Janssen, N., Kirschner, P. A., \& Erkens, G. (2015). Avoiding split attention in computer-based testing: Is neglecting additional information facilitative? British Journal of Educational Technology, 46(4), 803-817.

Just, M.A., \& Carpenter, P. (1976). Eye fixations and cognitive processes. Cognitive Psychology, 8(4), $441-480$.

de Koning, B. B. \& Jarodzka, H. (2017). Attention guidance strategies for supporting learning from dynamic visualizations. In R. Lowe \& R. Ploetzner (Hrsg.), Learning from dynamic visualization (S. 255-278). Cham: Springer International Publishing AG.

de Koning, B. B., Tabbers, H. K., Rikers, R. M., \& Paas, F. (2007). Attention cueing as a means to enhance learning from an animation. Applied Cognitive Psychology, 21, 731-746.

de Koning, B. B., Tabbers, H. K., Rikers, R. M., \& Paas, F. (2010). Attention guidance in learning from complex animation: seeing is understanding? Learning and Instruction, 20(2), 111-122.

Krapp, A. (1992). Konzepte und Forschungsansätze zur Analyse des Zusammenhangs von Interesse, Lernen und Leistung. In A. Krapp \& M. Prenzel (Hrsg.), Interesse, Lernen, Leistung (S. 9-52). Münster: Aschendorff.

Kriz, S., \& Hegarty, M. (2007). Top-down and bottom-up influences on learning from animations. International Journal of Human-Computer Studies, 65, 911-930.

Lindow, S., Fuchs, H.M., Fürstenberg, A., Kleber, J., Schweppe, J., \& Rummer, R. (2011). On the robustness of the modality effect: attempting to replicate a basic finding. Zeitschrift für Pädagogische Psychologie, 25(4), 231-243.

Loviscach, J. (2019). Inverted Classroom Model: mehr als nur eine Vorbereitung mit Videos. In S. Kauffeld \& J. Othmer (Hrsg.), Handbuch Innovative Lehre (S. 87-97). Wiesbaden: Springer.

Lowe, R. K., \& Schnotz, W. (2014). Animation principles in multimedia learning. In R. Mayer (Hrsg.), The Cambridge handbook of multimedia learning (S. 513-546). Cambridge: Cambridge University Press.

Mayer, R.E. (2014). The cambridge handbook of multimedia learning (2. Aufl.). Cambridge: Cambridge University Press.

Ozcelik, E., Arslan-Ari, I., \& Cagiltay, K. (2010). Why does signaling enhance multimedia learning? Evidence from eye movements. Computers in Human Behavior, 26(1), 110-117. 
Peeck, J. (1994). Wissenserwerb mit darstellenden Bildern. In B. Weidenmann (Hrsg.), Wissenserwerb mit Bildern (S. 59-94). Bern: Huber.

Persike, M. (2019). Denn sie wissen, was sie tun: Blended Learning in Großveranstaltungen. In S. Kauffeld \& J. Othmer (Hrsg.), Handbuch Innovative Lehre (S. 65-86). Wiesbaden: Springer.

Richter, J., \& Scheiter, K. (2019). Studying the expertise reversal of the multimedia signaling effect at a process level: evidence from eye tracking. Instructional Science, 47, 627-658.

Richter, J., Scheiter, K., \& Eitel, A. (2016). Signaling text-picture relations in multimedia learning: a comprehensive meta-analysis. Educational Research Review, 17, 19-36.

Rummer, R., Schweppe, J., Scheiter, K., \& Gerjets, P. (2008). Lernen mit Multimedia - Die kognitiven Grundlagen des Modalitätseffekts. Psychologische Rundschau, 59(2), 98-107.

Scheiter, K., \& Eitel, A. (2015). Signals foster multimedia learning by supporting integration of highlighted text and diagram elements. Learning and Instruction, 36, 11-26.

Schmidt-Weigand, F., Kohnert, A., \& Glowalla, U. (2010). Explaining the modality and contiguity effects: new insights from investigating students' viewing behaviour. Applied Cognitive Psychology, 24, 226-237.

Schneider, S., Beege, M., Nebel, S., \& Rey, G. D. (2018). A meta-analysis of how signaling affects learning with media. Educational Research Review, 23, 1-24.

Schöne, C., Dickhäuser, O., Spinath, B., \& Stiensmeier-Pelster, J. (2012). Skalen zur Erfassung des schulischen Selbstkonzepts - SESSKO (2. Aufl.). Göttingen: Hogrefe.

Schweinberger, M., \& Girwidz, R. (2017). Förderung angehender Physiklehrer durch die Arbeit mit stummen Videos. In C. Maurer (Hrsg.), Implementation fachdidaktischer Innovation im Spiegel von Forschung und Praxis (S. 680-683). Berlin: Springer.

Sweller, J., Ayres, P., \& Kalyuga, S. (2011). Cognitive load theory. New York: Springer.

Tabachnick, B. G., \& Fidell, L.S. (2014). Using multivariate statistics (6. Aufl.). Boston: Pearson.

Thomas, A., \& Müller, F.H. (2016). Entwicklung und Validierung der Skalen zur motivationalen Regulation beim Lernen. Diagnostica, 62(2), 74-84.

Xie, H., Mayer, R., Wang, F., \& Zhou, Z. (2019). Coordinating visual and auditory cueing in multimedia learning. Journal of Educational Psychology, 111(2), 235-255. 